\title{
Estado psicológico y estrategias educativas en docentes universitarios: en tiempos de pandemia Covid-19
}

\author{
Psychological status and educational strategies in university teachers:
}

\section{during Covid-19 pandemic}

1 Luis Guillermo Rosero

https://orcid.org/0000-0002-1508-8688 Universidad Técnica de Ambato. Carrera de Educación Básica. Tungurahua Ecuador. luisguillermorosero@uta.edu.ec

2 Víctor Daniel Aldás Rovayo

https://orcid.org/0000-0003-3315-2521 Universidad Estatal de Milagro, Carrera de Educación Especial, Guayas-Ecuador, valdasr@unemi.edu.ec

3 Willyams Rodrigo Castro Dávila iD https://orcid.org/0000-0002-2917-3461 Universidad Técnica de Ambato, Facultad de Ciencias Humanas y de la Educación, Carrera de Educación Básica, Ambato-Ecuador williamsrcastrod@uta.edu.ec

4 Héctor Manuel Neto Chusín (iD) https://orcid.org/0000-0002-8748-0064 Universidad Técnica de Ambato, Facultad de Ciencias Humanas y de la Educación, Carrera de Educación Básica, Ambato-Ecuador. hm.neto@uta.edu.ec

Artículo de Investigación Científica y Tecnológica Enviado: 24/12/2021 Revisado: 29/12/2021 Aceptado: 12/01/2022 Publicado:08/03/2023

DOI: https://doi.org/10.33262/concienciadigital.v6i1.4.2018

Cítese:

Guillermo Rosero, L., Aldás Rovayo, V. D., Castro Dávila, W. R., \& Neto Chusín, H. M. (2023). Estado psicológico y estrategias educativas en docentes universitarios: en tiempos de pandemia Covid-19. ConcienciaDigital, 6(1.4), 618-634. https://doi.org/10.33262/concienciadigital.v6i1.4.2018

\footnotetext{
CONCIENCIA DIGITAL, es una Revista Multidisciplinar, Trimestral, que se publicará en soporte electrónico tiene como misión contribuir a la formación de profesionales competentes con visión humanística y crítica que sean capaces de exponer sus resultados investigativos y científicos en la misma medida que se promueva mediante su intervención cambios positivos en la sociedad. https://concienciadigital.org

La revista es editada por la Editorial Ciencia Digital (Editorial de prestigio registrada en la Cámara Ecuatoriana de Libro con No de Afiliación 663) www.celibro.org.ec 


\section{Palabras} claves:

Covid-19, crisis sanitaria, estado psicológico y estrategias educativas
Keywords: Covid-19, health crisis, psychological status, and educational strategies.
Resumen

Introducción: El Covid-19 trajo un sinnúmero de consecuencias en las diversas áreas de la actividad humana, una de ellas en la educación universitaria; este fenómeno fue analizado bajo la óptica de los distintos actores educativos; sin embargo, el estudio se refiere al estado psicológico y a las estrategias educativas desde la perspectiva de los docentes de cuatro universidades de la Sierra ecuatoriana. Objetivo: el propósito es identificar el estado psicológico y las estrategias educativas utilizadas por los docentes universitarios en tiempos de crisis sanitaria. Método: la investigación es de tipo descriptiva correlacional de enfoque cuanti-cualitativa; el instrumento utilizado fue un cuestionario de veinte y ocho ítems, la primera parte corresponde al test del estado psicológico de Kessler y la segunda es un cuestionario sobre las estrategias metodológicas de la autoría de los investigadores con un valor estadístico de 0,73 del coeficiente de Alfa de Cronbach, aplicado a 70 docentes de las universidades: Técnica de Ambato, Central del Ecuador, Técnica de Cotopaxi, Estatal de Bolívar y Técnica del Norte. Resultados: los resultados muestran que el $61,76 \%$ de los profesores universitarios investigados padecen de malestar psicológico, entre ellos, el 33,82\% se ubica en el rango (22-29 ptos.) de intensidad alta y el $27,94 \%$ en (30-50 ptos.) que equivale a una intensidad muy alta; por otro lado, el $65 \%$ de los docentes utilizan recursos convencionales clásicos para la enseñanza, y con respecto a la educación mediada por herramientas tecnológicas a veces contribuyen al logro de los objetivos de aprendizaje. Conclusión: se identificó que más de la mitad de los docentes universitarios presentan síntomas de malestar psicológico, además continúan con el uso de estrategias educativas similares a las de la educación presencial y, por tanto, según la percepción de los propios profesores no se están alcanzando los resultados de aprendizaje esperados.

Introduction: Covid-19 brought countless consequences in many human activity areas like in university education. This phenomenon was analyzed from the point of view of different educational actors. However, the study refers to psychological status and educational strategies from the teachers' perspective from four universities in the Ecuadorian Sierra. Objective: The purpose is to identify the psychological status and educational strategies used by university 
teachers during a health crisis. Method: The research is a descriptive correlational type with a quantitative-qualitative focus. A survey of twenty-eight items was used, the first part corresponds to the Kessler test of psychological status and the second part is a survey about methodological strategies, its authorship corresponds to the researchers with a statistical value of 0,73 of the Cronbach's Alfa coefficient, applied to 70 teachers at the following universities: Technical University of Ambato, Central University of Ecuador, Technical university of Cotopaxi, Bolívar State University and Technical University of the North. Results: The results show that $61,76 \%$ of the university teachers investigated suffer from psychological discomfort, from that the $33,82 \%$ are in the range (22-29 pts.) of high intensity, and 27,94\% in (30-50 pts.) very high intensity. On the other side, $65 \%$ of teachers use classical conventional resources for teaching, and respect to education mediated by technological tools, sometimes contributes to achieving the learning objectives. Conclusion: It was identified that more than half of the university teachers register symptoms of psychological discomfort. Moreover, they continue using educational strategies like face-to-face education. Therefore, according to teachers' perception, they are not achieving the learning results expected.

\section{Introducción}

El coronavirus 2019 (Covid-19) es una enfermedad ocasionada por el virus del síndrome respiratorio agudo severo de tipo-2 (SARS-Cov-2) (Díaz y Toro, 2020). El virus SARSCov-2 produce la enfermedad denominada Covid-19 (Zuin et al., 2021). La pandemia apareció en diciembre de 2019 en Wuhan, provincia Hubei, China, en un mercado de comidas y de carne de animales silvestres; se transmitió y se extendió rápidamente por el resto de los continentes y en marzo de 2020 la Organización Mundial de la Salud (OMS) declaró la ocurrencia de la pandemia.

Los coronavirus desde el punto de vista epidemiológico se dividen en humanos y zoonóticos. La transmisión se da mediante contacto directo con gotitas de saliva, fómites aerosoles y superficies hasta por tres días con personas infectadas y asintomáticas; comienza de 1 a 2 días antes de los síntomas y afecta al $87 \%$ de la población en la edad de 30 a 79 años. La enfermedad en sí golpea más en los lugares en donde se colapsan los sistemas de salud pública, por el retraso en la atención primaria a los pacientes. La OMS define los casos de sospechoso, probable, confirmado y contacto. El periodo de 
incubación es de 2 a 7 días o incluso en 2 semanas con un período de cuarentena mínimo de 14 días.

Las medidas de prevención recomendadas son: distanciamiento social al menos dos metros y lavado frecuente de manos. Las entidades gubernamentales por esta razón determinaron el aislamiento obligatorio y cierre de fronteras como medida prioritaria (Díaz y Toro, 2020). El aislamiento social preventivo (cuarentena) obligatorio es una estrategia de control de la pandemia. Por estas razones, el confinamiento en el hogar trajo consigo un alto impacto en el bienestar físico y psicológico; en este último por la preocupación por los aspectos laborales, socioeconómicos y la salud mental (Zuin et al., 2021); a más de ello por la autopercepción negativa de envejecimiento en cualquiera de las edades, la ansiedad y tristeza en la población de mediana edad y en los jóvenes (Lozada et al., 2020); la ansiedad por el incremento de casos (Becerra et al., 2021) y por el tiempo de duración de las consecuencias psicosociales: trastornos en el estado de ánimo, somatoformo y estrés postraumático (Buitrago et al., 2020).

Las recomendaciones ante la pandemia son el fomento de medidas activas en la salud psicofísica integral: manejo de información, hábitos físicos saludables, apoyo psicológico y medidas de socialización rutinarias (Zuin et al., 2021); mayor necesidad de información sobre la ansiedad (Becerra et al., 2021) y una atención primaria de salud financiada, formada y reformada de ayuda conjunta a la población (Buitrago et al., 2020).

\section{Crisis sanitaria}

La crisis sanitaria es una amenaza o riesgo potencial que afecta a la población, ocurre cuando la toma de decisiones supera a la capacidad de respuesta. Los factores que aumentan la crisis son la detección tardía, el retraso en la aplicación de medidas de control, incertidumbre en la información y escasa confianza en las fuentes. La reducción del riesgo se alcanza al minimizar el tiempo entre el aparecimiento y la respuesta inicial. La detección, acceso y evaluación facilita la valoración oportuna de riesgos. En definitiva, la mejor herramienta para enfrentar es la comunicación acertada y transparente (Soria y Guillén, 2021).

El manejo de las emociones ante la crisis exige estrategias individuales, institucionales y sociales para reforzar la seguridad y reducir el temor (Brito et. al., 2020). Las medidas preventivas de mitigación son la reducción o supresión de ingresos hospitalarios y el avance en las investigaciones sobre la crisis (Abbas, 2021). El Instagram resultó ser el medio más idóneo para proporcionar información precisa y confiable (Malik et. al., 2021). La adopción de medidas frente la crisis sanitaria mediante actos administrativos restringe los derechos fundamentales a nivel individual o colectivo, por eso es necesario el control judicial que valore la proporcionalidad de la medida o una ley que regule la emisión de tales disposiciones (Sarrión Steve, 2020). 


\section{Malestar psicológico}

El malestar psicológico se define como el conjunto de síntomas que indican una alteración psicológica por la sobrecarga emocional, incomodidad, intranquilidad, ansiedad y depresión (Carranza et al., 2021). El malestar emocional y el burnout (agotamiento físico, mental o emocional) son el efecto del estrés laboral (Ovejas et al., 2020).

En el campo de la salud, un estudio con el personal de Perú mostró la correlación entre el nivel de malestar psicológico y la carga laboral, la preocupación por el contacto del Covid-19; y la cantidad de atenciones incrementaron la intranquilidad, nerviosismo, tristeza o desesperación (Carranza et al., 2021). El burnout y malestar psicológico en los residentes de medicina familiar y comunitaria de Barcelona evidenció porcentajes elevados de malestar psicológico y aún mayor el de burnout; estos valores se aumentaron en los últimos niveles de residencia (Ovejas, et al., 2020). Otra investigación sobre el impacto emocional de la pandemia de Covid-19 en trabajadores sanitarios en uno de los focos de contagio en Barcelona determinó los factores asociados al malestar emocional, el estar en contacto con pacientes de Covid-19, no haber realizado la PCR, la sensación de contar con elementos de protección y la muerte de un pariente cercano (Erquicia et al., 2020).

En tanto en el ámbito educativo, el estudio del malestar psicológico en estudiantes universitarios y posgrado de Guanajuato, México identificó los factores relacionados con la pandemia del Covid-19: prevalencia en los trastornos de ansiedad, estrés y depresión (Gutiérrez et al., 2021). Los resultados de la investigación en docentes de la escuela Miguel Paz de San Pedro, Honduras evidenciaron que la pandemia por el Covid-19 impactó en la salud emocional a través de pensamientos o sentimientos negativos: incertidumbre, frustración, tristeza y físico: dolores de cabeza y musculares (Fuentes y Zúniga, 2021). El trabajo con profesores de la Red Educativa Rural de las regiones de Huancavelica, Ica y Junín, Perú evidenció la presencia de malestares generales: irritabilidad, enfado, mal humor y problemas de sueño asociados al confinamiento (Quispe \& García, 2020). La mayor coincidencia entre estos estudios es la identificación de un alto nivel de malestar psicológico en las mujeres (Carranza et al., 2021; Erquicia et al., 2020 y Gutiérrez et al., 2021), ya sea por el incremento de las actividades laborales o domésticas en las que se vieron involucradas (Quispe y García, 2020).

\section{Estrategias educativas}

Ante esta situación, por un lado, según Ramos et al. (2018) los docentes se vieron obligados a la adopción de estrategias educativas emergentes y por otro, los modelos educativos actuales requieren de procesos de enseñanza - aprendizaje más activos, flexibles y dinámicos (Salazar et al., 2018). Las estrategias en sí son un sistema general de actividades dinámicas y flexibles que promueven un cambio cualitativo, tanto en el 
proceso como en los resultados (Correa et al., 2018); contribuyen a la calidad del aprendizaje mediante el logro de objetivos y el desarrollo de habilidades y destrezas (Gómez, 2017).

Murillo Vargas (2020) cita a Díaz y Hernández, quienes consideran a las estrategias de manera genérica como procedimientos (conjunto de acciones) para alcanzar un objetivo o resolver un problema a fin de adquirir conocimientos y se clasifican en estrategias de enseñanza, de aprendizaje y digitales. Las estrategias de enseñanza son acciones de apoyo docente para el procesamiento de la información por los aprendices; estas son preintruccionales, co-instruccionales y post-instruccionales; las pre-instruccionales (de inicio) se refieren a los objetivos, los organizadores previos, las señalizaciones y los conocimientos previos; las co-instrucionales (durante) aluden a ilustraciones, organizadores gráficos, preguntas intercaladas, mapas y redes conceptuales; y las postinstruccionales (término) se relacionan con la promoción de enlaces, resúmenes y analogías. Las estrategias de aprendizaje son un conjunto de acciones del estudiante para la articulación, integración y adquisición del conocimiento; son de ensayo, de elaboración, de control de la comprensión, de apoyo o efectivas y para el aprendizaje significativo. Las estrategias educativas digitales promueven el trabajo activo, colaborativo e interactivo entre educadores y educandos en la red.

Por otro lado, Díaz y Hernández (2010) definen a las estrategias desde la perspectiva del aprendizaje significativo como procedimientos o secuencia de acciones, actividades conscientes y voluntarias, técnicas $\mathrm{u}$ operaciones, habilidades empleadas de forma consciente, controlada e intencional; persiguen un propósito de aprendizaje a través de instrumentos que potencian el aprender a aprender. Estas estrategias la relacionan con los tipos de conocimiento que dan lugar a estrategias relacionadas con procesos cognitivos básicos, conocimientos conceptuales específicos, conocimiento estratégico y conocimiento metacognitivo. Los procesos cognitivos básicos son operaciones para el procesamiento de la información: atención, percepción, codificación, almacenamiento y recuperación; los conocimientos conceptuales específicos son los hechos, conceptos y principios referentes a los saberes previos; el conocimiento estratégico es el saber cómo conocer y; el metacognitivo es el conocimiento sobre el conocimiento, sobre qué y cómo se sabe.

Las estrategias de aprendizaje según este autor se clasifican en estrategias de recirculación de la información, de elaboración y organización; para el aprendizaje de contenidos declarativos de tipo factual: repetición simple, organización categorial y elaboración verbal y visual; para los contenidos declarativos complejos: representación gráfica de redes conceptuales, resumen de textos, elaboración conceptual y formulación de preguntas; y finalmente las estrategias referentes a la metacognición y regulación (Díaz y Hernández, 2010). 
La época actual exige la integración de las estrategias educativas con la tecnología digital para la mejora de la calidad del aprendizaje, con base en el pensamiento crítico (Murillo Vargas, 2020). El uso de los dispositivos móviles resulta una estrategia educativa renovada, por el fácil e inmediato acceso, ahorra la inversión de laboratorios y privilegian al estudiante como centro del proceso de enseñanza aprendizaje (Mangish y Mangish, 2019). Las redes sociales constituyen estrategias educativas que facilitan la comunicación y distribución de información, envío o entrega de tareas y clases en línea cuando el usuario no dispone de tiempo (Velasteguí López, 2018). En fin, el desarrollo del pensamiento computacional favorece la alfabetización digital, en tal sentido, es vital la generación de ecosistemas virtuales de aprendizaje que favorezcan la dimensión conceptual (saber), la aplicación (saber hacer) y la toma de decisiones (saber ser) para la resolución de problemas.

Además, se insiste en el uso estrategias cada vez más vinculadas a la realidad, el aprendizaje organizado por proyectos, los estudios de casos, la práctica in situ y el aprendizaje basado en problemas en cuanto estrategias educativas actuales que fomentan la formación en las competencias genéricas y específicas (Salazar et al., 2018). Los modelos pedagógicos insisten en la enseñanza de estrategias metacognitivas porque corresponden a procesos del pensamiento superior e influyen en la autonomía de los estudiantes y en nuevas perspectivas sobre la forma de evaluar a través del uso de diarios de aprendizaje (Díaz, 2015). En sí, la lectura es una estrategia de carácter universal y transversal que se ha configurado en los sistemas educativos como el medio idóneo para el aprendizaje por exploración propia; y la estrategia de "hojear, escanear y hacer predicciones" ayuda a la construcción del aprendizaje por sí mismo, promueve la rapidez, disfrute y desarrollo de habilidades para localizar información específica y general; y realizar predicciones sin depender directamente del profesor (Izquierdo \& Jiménez, 2014).

En definitiva, las estrategias se vinculan con procesos de formación en la medida que contribuyen al amor por la profesión, ayudar a crecer a los seres humanos y a mejorar las sociedades en búsqueda de un mundo más justo y humano. Existe una gran diferencia entre el educador y el transmisor de conocimientos; puesto que la educación a parte de ser ciencia o técnica es también arte. Es por ello por lo que, las estrategias requieren de pasión, experiencia y creatividad y no lo meramente técnico; estas provienen del corazón y no solo de la mente; están dispuestas para formar mejores personas (Gómez, 2017).

Es por esa razón, que el objetivo de este estudio es "Identificar el estado psicológico y las estrategias educativas utilizadas por los docentes universitarios en tiempos de crisis sanitaria" 


\section{Metodología}

El estudio está enmarcado desde un enfoque cuanti-cualitativo, con un alcance investigativo descriptivo- correlacional, estudio de caso aplicado a docentes de nivel superior de cinco universidades ecuatorianas. Se analizaron y se compararon variables demográficas, estrategias metodológicas y estado psicológico de los docentes universitarios. De la misma forma se realizó una investigación de campo, basado en la técnica de la encuesta, instrumento cuestionario auto aplicado.

La investigación se ejecutó en el primer semestre del año 2020; se consideró como población de estudio a 70 docentes universitarios de cinco instituciones de nivel superior del Ecuador, siendo las siguientes: Universidad Técnica de Ambato, Universidad Central del Ecuador, Universidad Técnica de Cotopaxi, Universidad Estatal de Bolívar y; Universidad Técnica del Norte. La selección de la muestra es de tipo no probabilística por voluntariado; estuvo conformada por 28 mujeres que representa el $40 \%$ y 42 hombres que representa el $60 \%$ de la población, además, la edad media de los encuestados es de 49.79 años (Mínimo=31 Máximo=69). Por otro lado, el tiempo de servicio o experiencia en docencia universitaria es de una media de 12.04 años.

Para la aplicación de los instrumentos se solicitó permiso institucional respectivo y el consentimiento informado de cada uno de los participantes, mismo que constaba el propósito u objetivo general de la investigación, el manejo ético y legal de la información entregada, como también el compromiso de proteger y cubrir la identidad en el momento de publicar los resultados investigativos. Además, la muestra estudiada fue obtenida por la voluntad, libre e independiente de los involucrados. No se consideró ningún tipo de retribución económica o de otra índole para quienes fueron parte del estudio.

Los instrumentos de recolección de datos fueron el test de malestar psicológico de Kessler $\mathrm{y}$, el cuestionario de estrategias educativas. Respecto al primer test se debe mencionar que se utilizó la adaptación al castellano por el grupo "Lisis" 2011, consta de 10 cuestiones específicas sobre el "malestar psicológico" de escala de Likert, cuenta con un nivel confiabilidad global de 0.90. El cuestionario de estrategias educativas fue de elaboración de los autores, misma que tuvo una validez de expertos en el tema, este contiene 23 ítems de tipo escala Likert, estimación de valores y preguntas dicotómicas. A su vez, mide las siguientes dimensiones: adaptabilidad a educación en línea, estrategias pedagógicas en la participación del estudiantado, tipo y medio de relación docente estudiante, recursos o herramientas de apoyo del docente, evidencia del aprendizaje, evaluación del proceso de enseñanza-aprendizaje, metodologías pedagógicas utilizadas $y$, requerimiento de apoyo curricular. Cabe indicar que la información fue recopilada mediante el uso de plataforma digital SurveyMonkey, el tiempo de auto aplicación promedio fue de $24 \mathrm{~min}$. 
Luego de realizar una revisión de la literatura científica respecto al estado psicológico y a las estrategias educativas de los docentes universitarios y su adaptación en tiempos de crisis sanitaria como también al impacto de la pandemia en el estado psicológico, se procedió a la aplicación de los test, de forma auto aplicada por la población en estudio. Posterior a la recogida de los datos se sometió al análisis y correlación de estos, especialmente en las variables sociodemográficas, estado psicológico y estrategias pedagógicas utilizadas, para ello se utilizó el software SPSS vs 24 , y finalmente, se estableció las conclusiones e interpretaciones respectivas.

\section{Resultados}

De acuerdo con la información recabada sobre los niveles de malestar psicológico en los docentes universitarios, se encontró que el $61.76 \%$ de los profesores universitarios investigados padecen de malestar psicológico, entre ellos, el 33.82\% se ubica en el rango (22-29 ptos.) de intensidad alta y; el 27.94\% se encuentra en el rango (30-50 ptos.) de intensidad muy alta (ver figura 1). Esto demuestra que un alto porcentaje del profesorado sufre los efectos colaterales de la pandemia, agravado por el cambio inadvertido de una educación presencial a virtual, además, por las responsabilidades de gestión y académicas que deben mantener los educadores de nivel superior.

\section{Figura 1}

Malestar psicológico en los docentes universitarios.

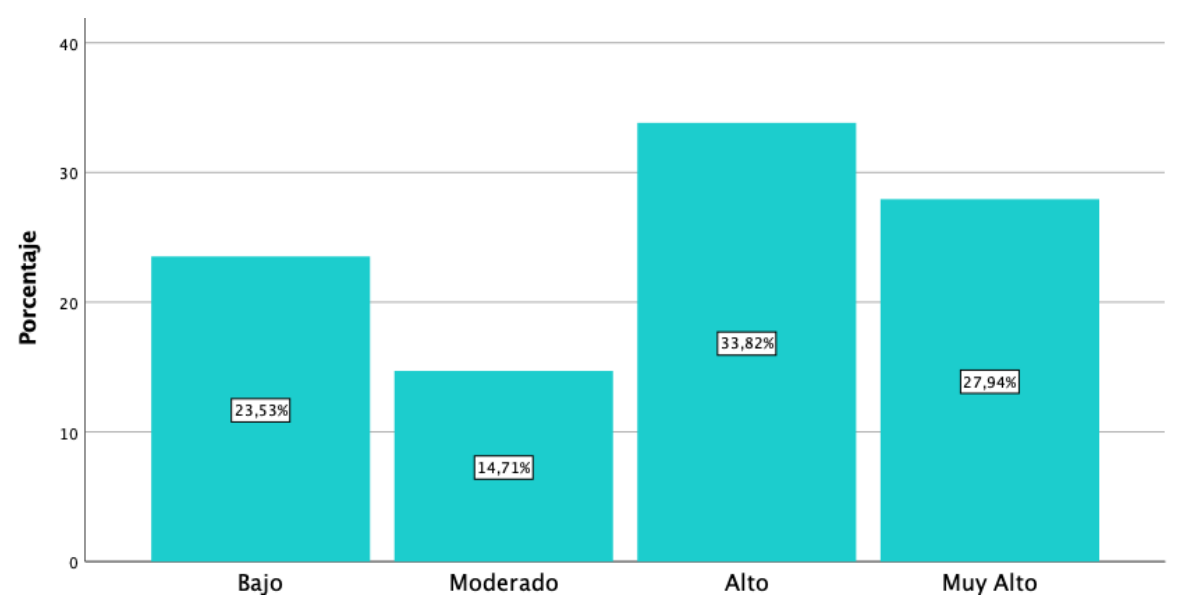

Nota: Niveles de malestar psicológico en relación con el porcentaje diagnosticado en docentes universitarios.

Fuente: Elaboración propia, aplicación del test de malestar psicológico de Kessler.

En este mismo sentido, a la hora de preguntarles a los docentes, si el tiempo en casa se transforma en tiempo de trabajo, se demostró que existe una diferencia significativa con relación a la variable sexo, es así como los 34 hombres que representa el 80.95\% refiere 
estar de acuerdo con la consigna dada, en tanto que las 15 mujeres que equivale al $53.57 \%$ manifiesta estar de acuerdo que el tiempo en casa se transforma en tiempo de trabajo.

A su vez, el porcentaje de diferencia entre hombres y mujeres en desacuerdo con la premisa establecida es muy significativa, siendo de $2.28 \%$ en hombres versus el $32.14 \%$ en mujeres (ver tabla 1).

Tabla 1

Tiempo de trabajo en casa según el sexo

\begin{tabular}{llrrr}
\hline & & \multicolumn{2}{c}{ Sexo } & \multicolumn{2}{c}{ Total } \\
\hline $\begin{array}{l}\text { El tiempo en casa } \\
\text { transforma en tiempo }\end{array}$ & seEn desacuerdo & Hombre & Mujer & 10 \\
trabajo & Ni de acuerdo ni en & $(2.28 \%)$ & $(32.14 \%)$ & 11 \\
& desacuerdo & 7 & 4 & 49 \\
& De acuerdo & $(16.67 \%)$ & $(14.29 \%)$ & 15 \\
\hline Total & & 34 & $(50.95 \%)$ & 70 \\
\hline
\end{tabular}

Fuente: Elaboración propia, aplicación del test de estrategias pedagógicas.

En la aplicación del estadístico Chi-cuadrado se comprueba que hay una relación entre las variables analizadas, con dos grados de libertad, y con un margen de error del 5\%, el valor de $\mathrm{p}$ es igual a 0.002 , resultando menor al esperado $(<\mathrm{p}=0,05)$ (ver tabla 2$)$.

\section{Tabla 2}

Pruebas de Chi-cuadrado, en relación con el tiempo de trabajo

\begin{tabular}{cccc}
\multicolumn{3}{c}{ en casa y sexo } \\
\hline & Valor & df & $\begin{array}{c}\text { Significación } \\
\text { asintótica } \\
\text { (bilateral) }\end{array}$ \\
\hline Chi-cuadrado de Pearson & $12.277 \mathrm{a}$ & 2 & .002 \\
Razón de verosimilitud & 12.935 & 2 & .002 \\
Asociación lineal por lineal & 10.156 & 1 & .001 \\
N de casos válidos & 70 & & \\
\hline
\end{tabular}

Nota: 2 casillas $(33,3 \%)$ han esperado un recuento menor que 5. El recuento mínimo esperado es 4,00. Fuente: Elaboración propia, análisis de datos en tablas cruzadas SPSS vs24.

En cuanto a la premisa de tener un equilibrio entre trabajo y familia, el personal académico en un $47.4 \%$ manifiesta estar en desacuerdo con tal enunciado, en tanto que el $35.7 \%$ está de acuerdo. Información que respalda un sentimiento de malestar psicológico, dado por los requerimientos y exigencias nuevas de trabajo y su interferencia con la convivencia familiar (ver figura 2). Según la literatura científica, la asociación en la vida 
social, afectiva y emocional entre trabajo y familia constituye en una de las causas más comunes de depresión y ansiedad patológica.

\section{Figura 2}

Equilibrio entre trabajo-familia

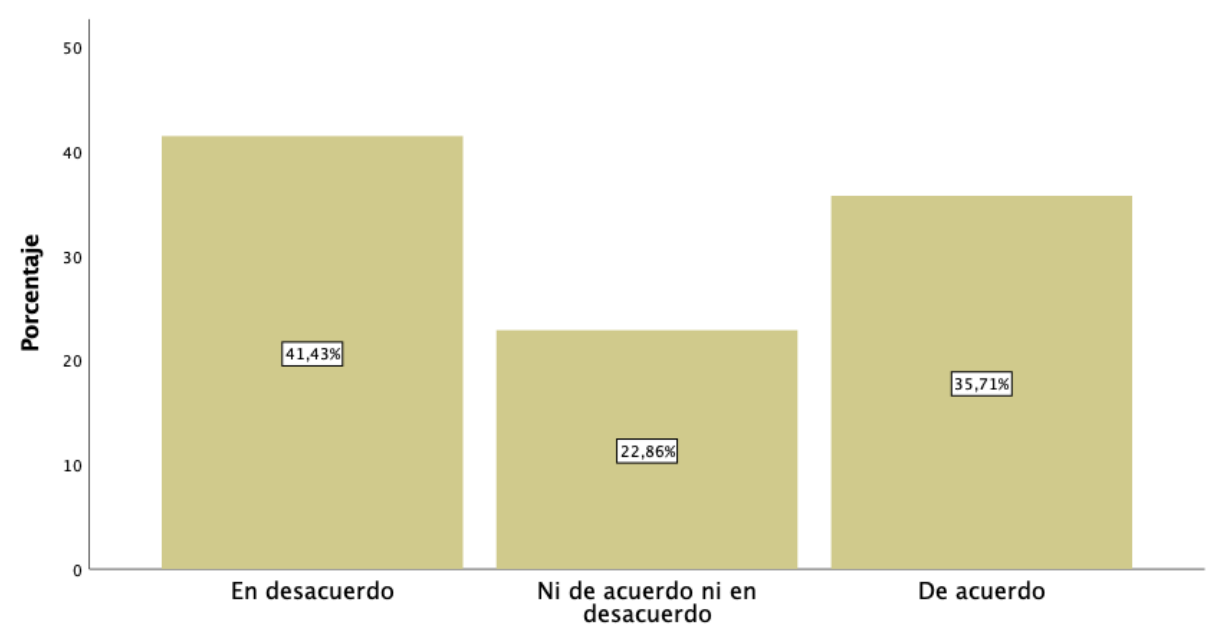

Nota: Relación porcentual de la variable trabajo y familia

Fuente: Elaboración propia, aplicación del test de malestar psicológico de Kessler y estrategias pedagógicas.

En cuanto a las estrategias pedagógicas se halló que el $82.9 \%$ de los docentes utilizan recursos convencionales clásicos para la enseñanza entre ellos están los documentos en formato Word, Pdf, PowerPoint; y multimedia (videos, clases pregrabadas y documentales); por otro lado, el $17.1 \%$ de los docentes utilizan los recursos poco convencionales, es decir, los interactivos de gamificación (ver tabla 3). Situación que concuerda con la necesidad de capacitación de los profesores en metodologías de aprendizaje activas mediadas por las tecnologías de la información y la comunicación.

Tabla 3

Recursos convencionales y no convencionales

\begin{tabular}{llrrrr}
\hline & Frecuencia & Porcentaje & $\begin{array}{c}\text { Porcentaje } \\
\text { válido }\end{array}$ & $\begin{array}{r}\text { Porcentaje } \\
\text { acumulado }\end{array}$ \\
\hline Válido & $\begin{array}{l}\text { Recursos } \\
\text { convencionales } \\
\begin{array}{l}\text { Recursos no } \\
\text { convencionales } \\
\text { Total }\end{array}\end{array}$ & 58 & 82.9 & 82.9 & 82.9 \\
& 70 & 17.1 & 17.1 & 100.0 \\
\hline
\end{tabular}

Fuente: Elaboración propia, aplicación del test de estrategias pedagógicas. 
De la misma forma, el tiempo empleado en las actividades en contacto con el docente mediado por herramientas de video conferencia en la impartición de clases es del $74 \%$ en promedio $(\min =25-\max =100)$, lo que demuestra mantener las mismas prácticas pedagógicas de la educación presencia. Tal desconocimiento u omisión de prácticas pedagógicas activas y adaptadas a la educación en línea, generan serios problemas en el avance académico de los estudiantes, además, limita la consecución de los objetivos planteados en cada asignatura.

Las actividades de aprendizaje que contemplan los docentes, con un grado de mayor a menor importancia son las siguientes: uno, se realiza procesos de observación de la realidad (40.82\%); dos, aplica transformadoramente los conocimientos (28.57\%); tres, ejecuta procesos de reflexión vinculando lo observado al conocimiento anterior (26.53\%), y cuatro, conceptualiza (4.08\%). Esto demuestra, la sintonía del proceso pedagógico con el método científico, partir de la observación de la realidad, reflexión o hipótesis del fenómeno, hasta llegar a la resolución o transformación de su contexto social.

Con respecto a la educación mediada por herramientas tecnológicas, los docentes universitarios refieren en su mayoría que a veces contribuyen al logro de los objetivos de aprendizaje en el 52.9\%, hecho que dificulta alcanzar las metas propuestas en el syllabus y el plan analítico de cada asignatura (ver tabla 4).

Tabla 4

Educación mediada por herramientas tecnológicas y logro de objetivos de aprendizaje

\begin{tabular}{cccccc}
\hline & Frecuencia & Porcentaje & $\begin{array}{c}\text { Porcentaje } \\
\text { válido }\end{array}$ & $\begin{array}{c}\text { Porcentaje } \\
\text { acumulado }\end{array}$ \\
\hline Válido & Nunca & 20 & 28,6 & 28,6 & 28,6 \\
& A veces & 37 & 52,9 & 52,9 & 81,4 \\
& Siempre & 13 & 18,6 & 18,6 & 100,0 \\
& Total & 70 & 100,0 & 100,0 & \\
\hline
\end{tabular}

Fuente: Elaboración propia, aplicación del test de estrategias pedagógicas.

Cabe mencionar, que el acceso a la internet y dispositivos electrónicos como computador, laptop o celular es limitado para los estudiantes de pocos recursos económicos o en los que viven en zonas rurales. Esto dificultad más la adquisición de competencias y habilidades profesionales.

\section{Conclusiones}

- Casi la tercera parte de los docentes consultados consideran que en el trabajo durante la pandemia han experimentado un malestar psicológico, principalmente 
por el repentino cambio de modalidad, sin una preparación previa y adaptación acelerada ante una incierta realidad; un traslado brusco de lo presencial a lo virtual se tuvo que asumir a costa de grandes cambios, adecuaciones en el hogar y retos para llevar adelante la nueva forma de laborar.

- En referencia al equilibrio que mantienen los docentes entre las actividades laborales y la familia, casi la mitad de los consultados, manifiestan estar en desacuerdo, por cuantos los horarios de trabajo fueron más allá de los habituales, resultó difícil sobrellevar las tareas académicas y de gestión docente y las responsabilidades familiares al mismo tiempo.

- En lo referente a las estrategias educativas utilizadas por docentes en la nueva modalidad de trabajo se encontró que el $82.9 \%$ de los profesores continúan con el uso de recursos convencionales clásicos para la enseñanza, entre ellos están los documentos en formato Word, Pdf, PowerPoint; y multimedia (videos, clases pregrabadas, documentales); y un mínimo porcentaje, el $17.1 \%$ del profesorado utilizan los recursos no convencionales mediadas por las tecnologías de la información y comunicación, por ejemplo las tareas interactivas como la gamificación.

\section{Referencias Bibliográficas}

Abbas, J. (2021, 4 de febrero). Gestión de crisis, desafíos y oportunidades sanitarios transnacionales: la intersección de la pandemia de COVID-19 y la salud mental global. Investigación en globalización, 3(2021), 1-7. https://doi.org/10.1016/j.resglo.2021.100037

Becerra-García, J. A., Sánchez-Gutiérrez, T., \& Barbeito, S. y Calvo, A. (2021, 15 de mayo). Pandemia por COVID-19 y salud mental en España: un análisis de su relación utilizando Google Trends. Revista de Psiquiatría y Salud Mental, 14(3), 1-8. https://doi.org/10.1016/j.rpsm.2021.05.001

Brito-Brito, P. R., Fernández-Gutiérrez, D. A., \& Cuéllar-Pompa, L. (2020, 11 de mayo). Manejo emocional Delaware la crisis sanitaria apuesta inicial el coronavirus: una revisión narrativa. Enfermería clínica, 31(1), 107-111. https://doi.org/10.1016/j.enfcli.2020.05.010

Buitrago-Ramírez, F., Ciurana-Misol, R., Fernández-Alonso, M. C., Tizón-García, J. L. \& Grupo de Salud Mental del PAPPS. (2020, 30 de diciembre). Salud mental en epidemias: una perspectiva desde la Atención Primaria de Salud española. Atención Primaria, 52(2), 93-113. https://doi.org/10.1016/j.aprim.2020.09.004

Carranza-Estevan, R. F., Mamani-Benito, O. J., Quinteros-Zúniga, D., \& Farfán-Solís, R. (2021, 14 de junio). Preocupación por el contagio de la COVID-19 y carga laboral 
como predictores del malestar psicológico durante la emergencia sanitaria en personal de salud de Perú. Revista Colombiana de Psiquiatría, 1-7. https://doi.org/10.1016/j.rcp.2021.06.005

Correa-Contento, R. A., Guamán-Coronel, M. A., \& Beltrán-Guevara, P. M. (2018, 1 de abril). Estrategia educativa para potenciar los aprendizajes en los estudiantes de séptimo año de la Unidad Educativa Fiscomisional "La Dolorosa" de la ciudad de Loja. Revista Electrónica Entrevista Académica, 1(2), 301-313. https://bit.ly/3EAdSZa

Díaz, Í. (2015, 20 de octubre). Entrenamiento de estrategias metacognitivas para mejorar vocabulario a través de diarios de aprendizaje. PROFILE, 17(1), 87-102. https://doi.org/10.15446/profile.v17n1.41632

Díaz-Castrillón, F. J. \& Toro-Montoya, A. I. (2020, 26 de abril). SARS-CoV-2/COVID19: el virus, la enfermedad y la pandemia. Medicina y Laboratorio, 24(3), 183205. https://doi.org/10.36384/01232576.268

Díaz-Barriga, F. \& Hernández-Rojas, G. (2010). Estrategias docentes para un aprendizaje significativo, una interpretación constructivista (Vol. Tercero). México: McGraw-Hill.

Erquicia, J., Valls, L., Barja, A., Gil, S., Miquel, J., Leal-Blanquet, J., Schmidt, Checa, J. \& Vega, D. (2020, 20 de noviembre). Impacto emocional de la pandemia de Covid-19 en los trabajadores sanitarios de uno de los focos de contagio más importantes de Europa. Medicina Clínica, 155(10), 434-440. https://doi.org/10.1016/j.medcli.2020.07.006

Fuentes-Gómez, R. A. \& Zúniga-Valle, C., M. (2021, 21 de junio). Impacto psicológico en los docentes de educación primaria a causa de la covid-19. Academo, 8(2), 129139. https://doi.org/10.30545/academo.2021.jul-dic.2

Gutiérrez-García, R. A., Amador-Licona, N., Sánchez-Ruíz, A., \& Fernández-Reyes, P. L., (2021, 21 de marzo). Malestar psicológico, medidas sanitarias y estado de salud en estudiantes universitarios. Nova Scientia, 13, 1-17. https://doi.org/10.21640/ns.v13ie.2602

Gómez-Galán, J. (2017, mayo). Educational research and teaching strategies in the digital society: a critical view. Universidad de Extremadura y Universidad Metropolitana, 105-119. https://bit.ly/3pOCucF

Izquierdo-Castillo, A., \& Jiménez-Bonilla, S. (2014, octubre). Formación en autonomía a través de estrategias de lectura. Profile, 16(2), 67-85. http://dx.doi.org/10.15446/profile.v16n2.39904 
Lozada-Baltar, A., Márquez-González, M., Jiménez-Gonzalo, L., Pedroso-Chaparros, M. S., Gallego-Alberto, L. \& Fernández-Pires, J. (2020, 4 de junio). Diferencias en función de la edad y la autopercepción del envejecimiento en ansiedad, tristeza, soledad y sintomatología comórbida ansioso-depresiva durante el confinamiento por la COVID-19. Revista Española de Geriatría y Gerontología, 55(5), 272-278. https://doi.org/10.1016/j.regg.2020.05.005

Malik, A., Khan, M. L., \& Quian-Haase, A. (2021, 27 de mayo). Difusión de agencias de salud pública a través de Instagram durante la pandemia COVID-19: perspectiva de comunicación de riesgos de crisis y emergencias. Internacional diario de desastre Riesgo Reducción, 61, 1-9. https://doi.org/10.1016/j.ijdrr.2021.102346

Mangish-Moyano, G. C., \& Mangish-Spinelli, M. R. (2019, 31 de agosto). El uso de dispositivos móviles como estrategia educativa en la universidad. Revista Iberoamericana de Educación a Distancia, 23(1), 201-216. https://doi.org/10.5944/ried.23.1.25065

Murillo-Vargas, G. (2020). Estrategias educativas y tecnología digital en el proceso de enseñanza aprendizaje. Revista Cuadernos, 61(1), 69-76. https://bit.ly/3GeuJSM

Ovejas-López, A., Izquierdo, F., Rodríguez-Barragán, M., Rodríguez-Barragán, J., García-Batanero, M., Alonso-Martínez, M., \& Alonso-Masanas, C. (23 de junio de 2020). Burnout y malestar psicológico en los residentes de Medicina Familiar y Comunitaria. Atención primaria, 52(9), 608-616. https://doi.org/10.1016/j.aprim.2020.02.014

Quispe-Victoria, F. \& García-Curo, G. (2020, 20 de octubre). Impacto psicológico del covid-19 en la docencia de la Educación Básica Regular. Alpha Centauri, 01(02), 30-41. https://doi.org/10.47422/ac.v1i2.10

Salazar, C. M, Peña-Vargas, C. S. \& Medina-Valencia, R. T. (2018). Estrategias de enseñanza y aprendizaje para la docencia universitaria. Experiencias desde el aula. Universidad de Colima. https://bit.ly/3n9oW9R

Sarrión, J. (2020, 14 de septiembre). La competencia Delaware las autoridades sanitarias paraca restringir derechos en situación Delaware crisis sanitaria. Gaceta Sanitaria, 6(35), 1-4. 10.1016/j.gaceta.2020.07.009

Velasteguí-López, E. (05 de octubre de 2018). El uso de las redes sociales como estrategia educativa. $\quad$ Explorador digital, $\quad$ 2(4), https://doi.org/10.33262/exploradordigital.v2i4.341 
Soria, S. F. \& Guillén-Enríquez, F. J. (2008, 4 de febrero). Amenazas para la salud y crisis sanitarias. Aproximación a la alerta y respuesta. Informe SESPAS 2008. Gaceta Sanitaria, 22(suplemento 1), 65-70. $\quad$ https://doi.org/10.1016/S02139111(08)76076-X

Zuin, D. R., Zuin, M. P., Peñalver, F. \& Tarrulla, A. (2021, 20 de agosto). Pandemia COVID 19: aspectos psicológicos, alteraciones conductuales y perfil de hábitos. Resultados de una encuesta en el ámbito universitario a un mes del confinamiento total en Mendoza Argentina. Neurología Argentina, 13(4), 228-237. https://doi.org/10.1016/j.neuarg.2021.08.005

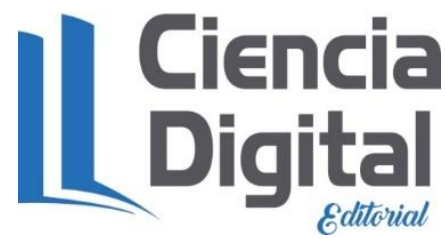


El artículo que se publica es de exclusiva responsabilidad de los autores y no necesariamente reflejan el pensamiento de la Revista Conciencia Digital.

\section{Ciencia}

El artículo queda en propiedad de la revista y, por tanto, su publicación parcial y/o total en otro medio tiene que ser autorizado por el director de la Revista Conciencia Digital.
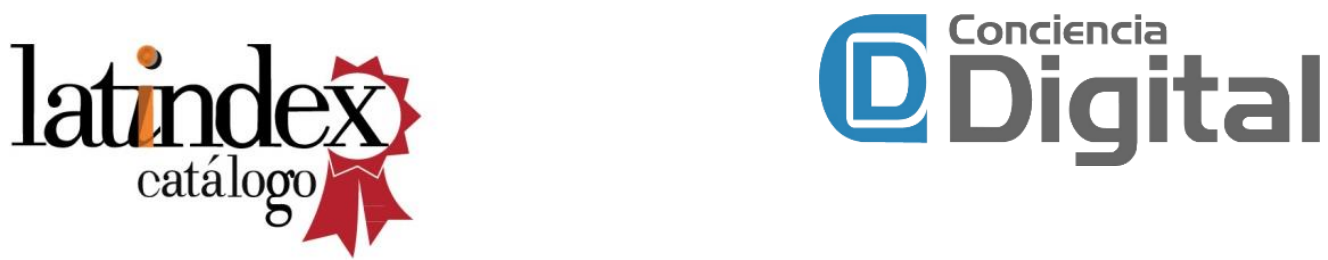

Indexaciones

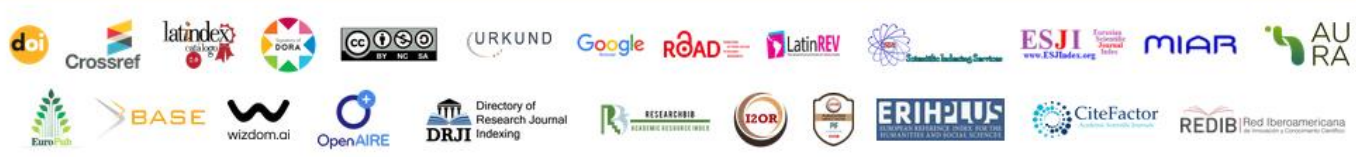

